\title{
Phase Diagram and Dielectric Relaxation Studies of $n$-Hexyl-isothiocyanato-biphenyl (6BT) in the Smectic E Phase under High Pressure
}

\author{
Stanislaw Urban and Albert Würflinger ${ }^{\mathrm{a}}$ \\ Institute of Physics, Jagellonian University, Reymonta 4, PL-30-059 Cracow, Poland \\ ${ }^{a}$ Physical Chemistry II, Ruhr-University Bochum, D-44780 Bochum \\ Reprint requests to Prof. S. U.; Fax: 0048-12-6337086; E-mail: ufurban@cyf-kr.edu.pl \\ Z. Naturforsch. 57 a, 233-236 (2002); received February 25, 2002
}

\begin{abstract}
The pressure-temperature phase diagram of $n$-hexyl-isothiocyanato-biphenyl (6BT) at up to $190 \mathrm{MPa}$ and $250-400 \mathrm{~K}$ was established with the aid of DTA. The dielectric relaxation measurements in the SmE phase of 6BT were performed in the pressure range of $0.1-150 \mathrm{MPa}$ and the temperature range of $320-350 \mathrm{~K}$. The Debye-type relaxation process was observed in the frequency range of $100 \mathrm{~Hz}-100 \mathrm{kHz}$. The longitudinal relaxation time $\tau$ characterizing the molecular reorientations around the short axis was analyzed with respect to the pressure and temperature dependencies, yielding the activation volume, $\Delta^{\#} V=R T(\partial \ln \tau / \partial p)_{T}$, and activation enthalpy, $\Delta^{\#} H=R\left(\partial \ln \tau / \partial T^{-1}\right)_{p}$, respectively. The results are compared with the analogous data obtained recently for $8 \mathrm{BT}$ and other similar compounds having the nematic and SmA liquid crystalline phases.
\end{abstract}

Key words: Liquid Crystals; Smectic E; DTA; Phase Diagram; Dielectric Relaxation; High Pressure.

\section{Introduction}

The $n$-alkyl-biphenyl compounds with the -NCS group in the terminal position $(n \mathrm{BTs})$ exhibit exclusively the smectic E (SmE) polymorphism [1], contrary to the well known substances with the $\mathrm{CN}$ group $(n \mathrm{CBs})$, which form the nematic $(\mathrm{N})$ and / or smectic $\mathrm{A}_{d}\left(\mathrm{SmA}_{d}\right)$ phases. Recently we have studied the octyl homologue, 8BT, under high pressure (DTA, $p V T$ and dielectric relaxation $[2,3]$ ). The DTA measurements have shown that the clearing line splits above ca. $190 \mathrm{MPa}$. The results of the dielectric relaxation studies yielded information about the influence of pressure on the parameters characterising the molecular rotations around the short axes in the SmE phase (relaxation time, activation volume and enthalpy). These quantities have shown some peculiar properties which were discussed in relation to the results obtained for other liquid crystals. The aim of the present study was to find whether these features are also observed for the shorter hexyl homologue, 6BT.

\section{Experimental}

The 6BT sample was the same as in [1]. The DTA measurements were carried out in the same way as for $8 \mathrm{BT}$ [2] with the use of two high-pressure devices [4]. The SmE phase of $6 \mathrm{BT}$, like that of $8 \mathrm{BT}$, showed a strong tendency to supercool, thereby forming metastable crystal phases. In order to obtain the stable crystal phase, the sample was annealed for a long time at low temperature (ca. $-30{ }^{\circ} \mathrm{C}$ ). However, even quick pressure changes as applied in [2] did not guarantee reproducible melting temperatures. The DTA traces revealed a rather small heat effect, contrary to the melting of 8BT [2].

The dielectric relaxation studies of $6 \mathrm{BT}$ in the $\mathrm{SmE}$ phase were performed with the use of the high pressure set-up in the Bochum University [5-9]. The complex dielectric permittivity, $\varepsilon^{*}=\varepsilon^{\prime}-i \varepsilon^{\prime \prime}$, was measured with an HP 4192A impedance analyzer in the frequency range of $100 \mathrm{~Hz}-100 \mathrm{kHz}$. However, below ca. $300 \mathrm{~Hz}$ the spectra showed a large scatter of points which limited the frequency range for the 


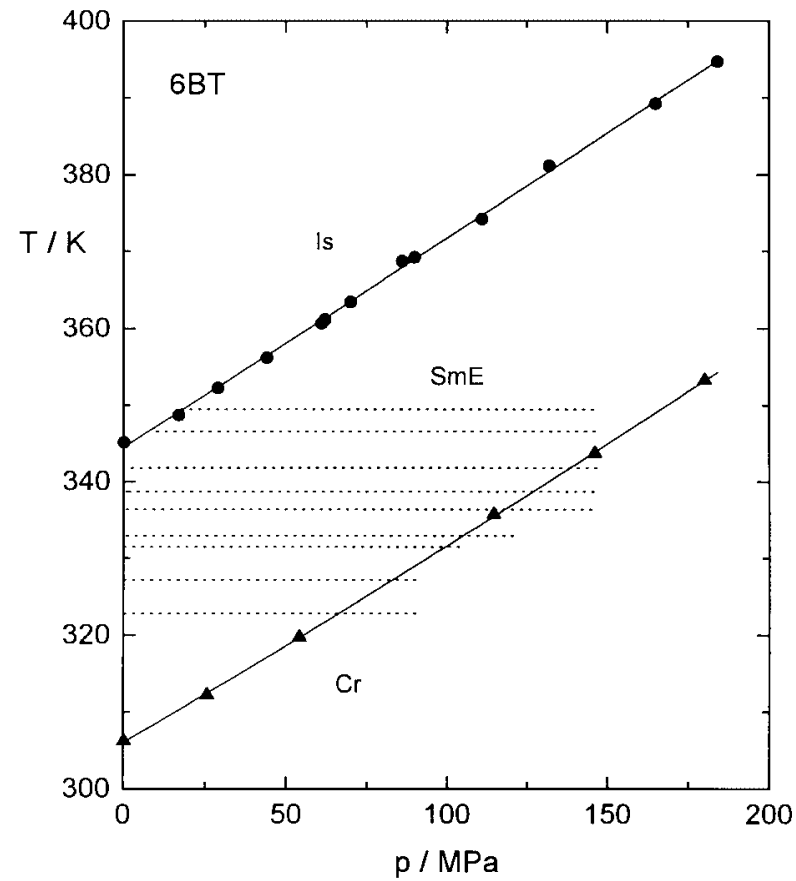

Fig. 1. Pressure-temperature phase diagram of 6BT established with DTA. Dotted lines mark the temperatures at which the dielectric relaxation studies were performed.

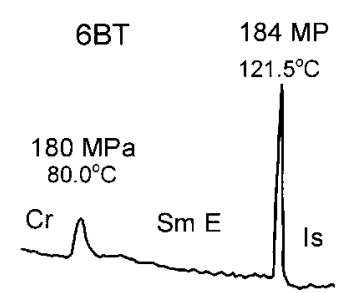

Fig. 2. A typical DTA trace on heating for 6BT.

final evaluations. The temperature was stabilized within $\pm 0.2 \mathrm{~K}$.

\section{Results}

Figure 1 presents the pressure-temperature phase diagram of 6BT. The pressure dependence of the transition temperatures can be expressed by the equations ( $T$ in $\mathrm{K}, p$ in $\mathrm{MPa}$ )

$$
\begin{aligned}
& T_{\mathrm{cl}}=344.7+0.266 p-0.34 \cdot 10^{-4} p^{2}, \\
& T_{\mathrm{m}}=306.0+0.249 p-0.73 \times 10^{-4} p^{2} .
\end{aligned}
$$

A typical DTA trace is shown in Figure 2. The small and not very sharp melting peak might indicate that

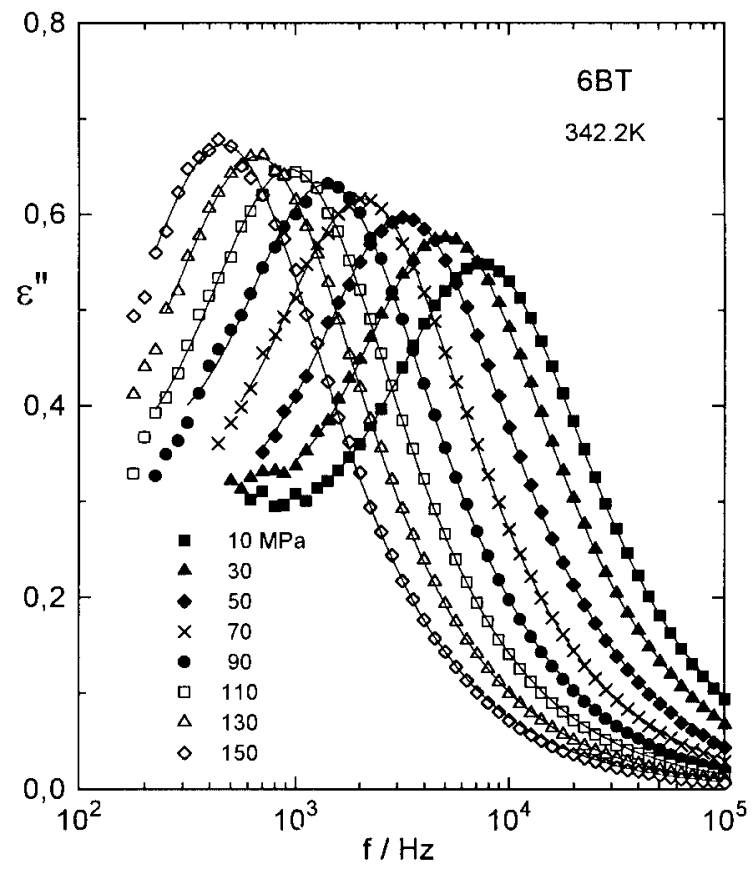

Fig. 3. Examples of the absorption spectra measured at one isotherm $(342.2 \mathrm{~K})$ and several pressures. The lines are fits of the Debye equation.

the preceding crystallisation process was not yet completed (in the case of 8BT [2] both peaks had comparable heights).

Typical absorption spectra measured in the SmE phase at constant temperature and different pressures are shown in Figure 3. The well known Cole-Cole modification of the Debye equation, $\left(\varepsilon^{*}-\varepsilon_{\infty}\right) /\left(\varepsilon_{\mathrm{s}}-\right.$ $\left.\varepsilon_{\infty}\right)=1 /(1+i \omega \tau)^{1-\alpha}$, was fitted to the experimental points $\left(\varepsilon_{\mathrm{s}}\right.$ and $\varepsilon_{\infty}$ are the static and high frequency permittivities, respectively, $\omega=2 \pi \nu, \tau$ is the relaxation time). The parameter $\alpha$, characterizing the distribution of the relaxation times, is very small (ca. 0.03 - 0.05), so we may consider the observed relaxation process as the Debye process. The static permittivity in the SmE phase $(4.0 \div 4.8)$ is markedly lower than the isotropic value (6.5 [1]). It is due to a lack of alignment of the sample; neither a magnetic nor electric field influenced the permittivity in the SmE phase of 6BT. However, there is no doubt that the relaxation spectra reflect the molecular reorientations around the short axes, because the dipolar group (NCS) gives a pronounced contribution in the direction of the long molecular axis.

The longitudinal relaxation times $\tau_{1}$, calculated from the fits of the imaginary part of the Debye 


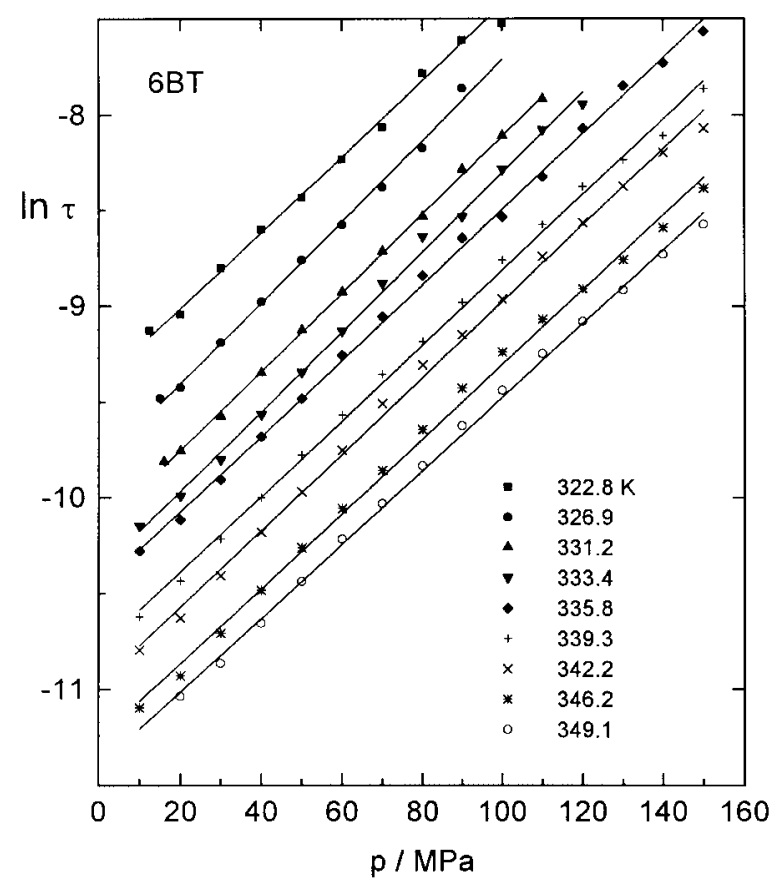

Fig. 4. Logarithm of the longitudinal relaxation time versus pressure for all isotherms studied within the SmE phase of 6BT. The straight lines fit the points that yields the activation volume.

equation to the absorption spectra measured at different temperatures and pressures are presented in Fig. 4 in semilogarithmic scale. The points for a given $T$ can nicely be approximated by straight lines. The slopes of the lines give the activation volumes $\Delta^{\#} V(T)=R T(\partial \ln \tau / \partial p)_{T}$ which are presented in Figure 6a. Taking the data in Fig. 4 at constant pressures enabled us to draw the Arrhenius plots (Fig. 5); their slopes yield the activation enthalpies $\Delta^{\#} H(p)=$ $R\left(\partial \ln \tau / \partial T^{-1}\right)_{p}$ that are shown in Figure $6 \mathrm{~b}$.

\section{Discussion}

In $[1,2,10]$ it has been shown that the parameters characterising the molecular rotations around the short axes $\left(\tau_{1}, \Delta^{\#} V, \Delta^{\#} H\right)$ change differently at the clearing temperature. The longitudinal relaxation time $\tau_{1}$ depends sensitively on whether the transition to the liquid-like $(\mathrm{N}, \mathrm{SmA}, \mathrm{C})$ or to the crystal-like $(\mathrm{SmB}, \mathrm{G}, \mathrm{E}, \ldots)$ phases occurs. The $\tau$ values taken at the clearing temperatures differ considerably between the liquid-like and crystal-like phases: $\tau^{6 \mathrm{CB}}=24.5 \mathrm{~ns}$, $\tau^{6 \mathrm{CHBT}}=13.5 \mathrm{~ns}, \tau^{6 \mathrm{DBT}}=20.8 \mathrm{~ns}$, whereas $\tau^{6 \mathrm{BT}}=$ $12530 \mathrm{~ns}$ ! The so-called retardation factor $g=\tau_{\mathrm{LC}} / \tau_{\text {is }}$

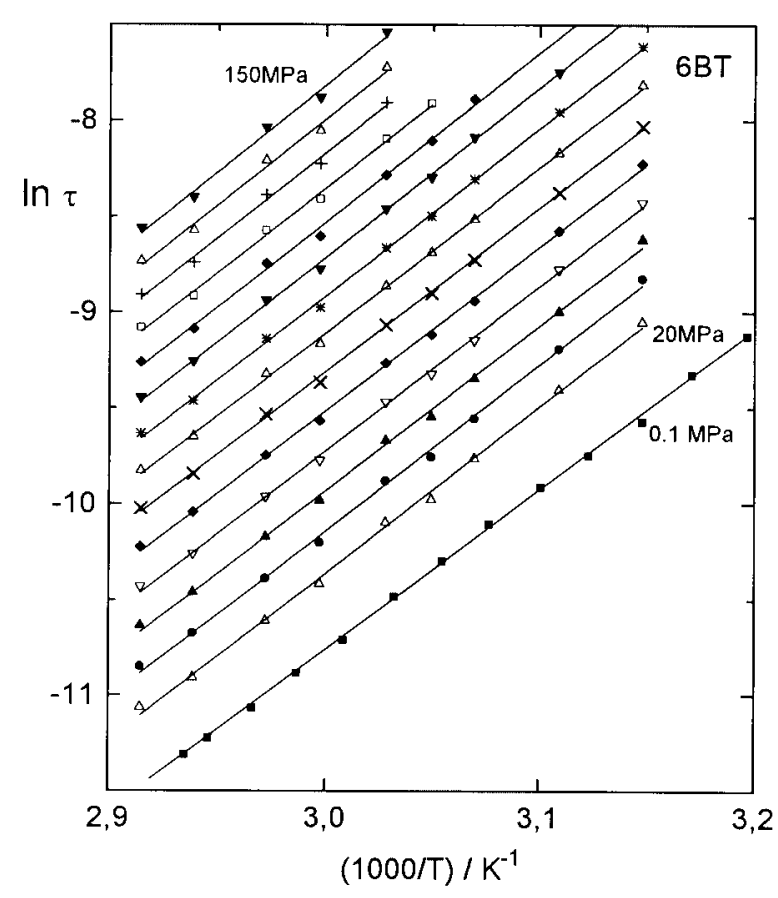

Fig. 5. Arrhenius plots for several isobars (in steps of $10 \mathrm{MPa}$ between 20 and $150 \mathrm{MPa}$ ) within the SmE phase of $6 \mathrm{BT}$ that yields the activation enthalpy. The data at atmospheric pressure were taken from [1].

is small (ca. $3 \div 10$ ) in the former case, whereas it is of the order of $10^{4}$ in the latter case. In contrast to that, the activation parameters are almost equal in both types of LC phases. Figure 6 shows this feature also for the hexyl members of four two-ring homologous series: 6CB [7], 6CHBT [8], 6DBT [11], and 6BT. As usually, the activation parameters determined for the SmA phase are smallest. Unlike to the cyano-compounds $[4,6,12]$, the activation parameters obtained for the isothiocyanato substances do not diminish with rising $T\left(\Delta^{\#} V\right)$ or $p\left(\Delta^{\#} H\right)$, see Figure 6 . On the other hand, the activation enthalpy slightly increases with $p$ for both $n \mathrm{BTs}$, that could be caused by the decreasing intermolecular distance with rising pressure. This would correspond to the observation that the dipoledipole correlations responsible for the effects in the cyano-compounds are absent in the substances with the NCS group. However, the small pressure effect seen in Fig. 6 should not be overestimated (taking into account the experimental errors), since a detailed volumetric study of 8BT did not yield an increase of the isochoric activation energy with increasing density [3]. 

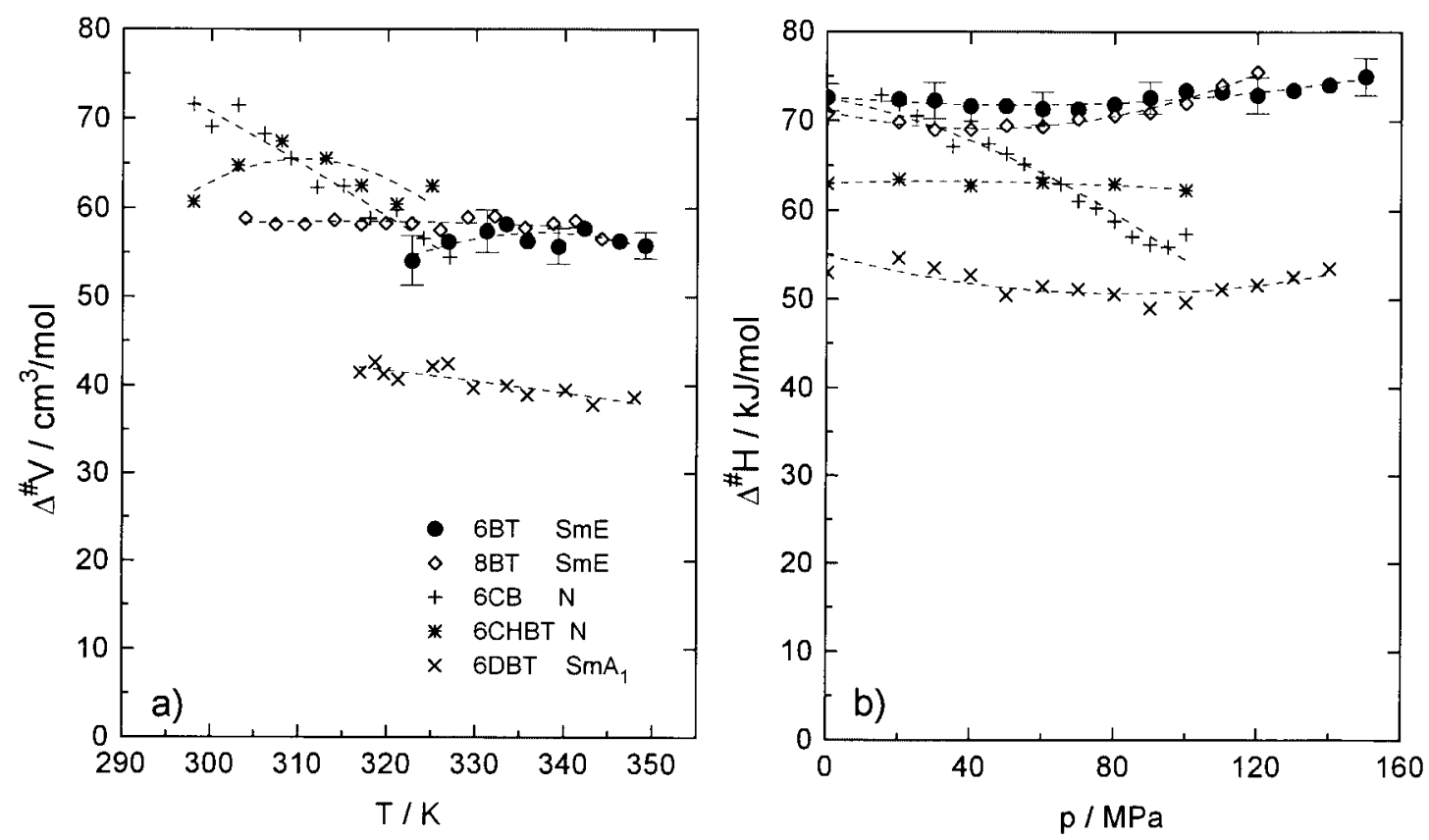

Fig. 6. Comparison of the activation volumes (a) and activation enthalpies (b) in different LC phases for several hexyl two-ring compounds. The lines are guides for the eye only.

\section{Acknowledgements}

The presented work was supplied by The Project for Bilateral Cooperation in Science and Technology, POL 99/011, sponsored by DLR (Germany) and KBN (Poland), and in part by the KBN Grant No 2 P03B 05222.

[1] S. Urban, K. Czupryński, R. Dąbrowski, B. Gestblom, J. Janik, H. Kresse, and H. Schmalfuss, Liq. Cryst. 28, 691 (2001).

[2] S. Urban, A. Würflinger, and A. Kocot, Liq. Cryst. 28, 1331 (2001).

[3] A. Würflinger and S. Urban, Liq. Cryst. 29, (2002), in press.

[4] N. Pingel, U. Poser, and A. Würflinger, J. Chem. Soc. Faraday Trans. I 80, 3221 (1984); C. Schmidt, M. Rittmeier-Kettner, H. Becker, J. Ellert, R. Krombach, and G. M. Schneider, Thermochim. Acta 238, 321 (1994).

[5] U. Poser and A. Würflinger, Ber. Bunsenges. Phys. Chem. 92, 765 (1988).

[6] S. Urban and A. Würflinger, Adv. Chem. Phys. 98, 143 (1997).

[7] S. Urban, T. Brückert, and A. Würflinger, Liq. Cryst. 15, 919 (1993).

[8] S. Urban and A. Würflinger, Liq. Cryst. 12, 931 (1992).

[9] S. Urban and A. Würflinger, Z. Naturforsch. 54a, 455 (1999).

[10] S. Urban, B. Gestblom, and R. Dabrowski, Polish J. Chem. 76, 263 (2002).

[11] A. Würflinger and S. Urban, Phys. Chem. Chem. Phys. 3, 3727 (2002).

[12] T. Brückert, D. Büsing, S. Urban, and A. Würflinger, in: Liquid Crystals: Physics, Technology and Applications, Proceed. of SPIE, 3318, 198 (1998). 